\title{
Equitable coloring of graphs. Recent theoretical results and new practical algorithms
}

\author{
HANNA FURMAŃCZYK, ANDRZEJ JASTRZĘBSKI and MAREK KUBALE
}

\begin{abstract}
In many applications in sequencing and scheduling it is desirable to have an underlaying graph as equitably colored as possible. In this paper we survey recent theoretical results concerning conditions for equitable colorability of some graphs and recent theoretical results concerning the complexity of equitable coloring problem. Next, since the general coloring problem is strongly NP-hard, we report on practical experiments with some efficient polynomial-time algorithms for approximate equitable coloring of general graphs.
\end{abstract}

Key words: computer experiments, corona graph, equitable chromatic number, equitable coloring conjectures, NP-hardness, polynomial heuristics.

\section{Introduction}

All graphs considered in this paper are finite and simple, i.e. undirected, loopless and without multiple edges.

If the set of vertices of a graph $G$ can be partitioned into $k$ (possibly empty) classes $V_{1}, V_{2}, \ldots, V_{k}$ such that each $V_{i}$ is an independent set and the condition ||$V_{i}|-| V_{j}|| \leqslant 1$ holds for every pair $(i, j)$, then $G$ is said to be equitably $k$-colorable. The smallest integer $k$ for which $G$ is equitably $k$-colorable is known as the equitable chromatic number of $G$ and is denoted by $\chi_{=}(G)$ [30]. Since equitable coloring is a proper coloring with an additional constraint, we have $\chi(G) \leqslant \chi_{=}(G)$ for any graph $G$.

This model of graph coloring has many practical applications. Every time when we have to divide a system with binary conflict relations into equal or almost equal conflictfree subsystems we can model this situation by means of equitable graph coloring. One motivation for equitable coloring suggested by Meyer [30] concerns scheduling problems. In this application, the vertices of a graph represent a collection of tasks to be

H. Furmańczyk, the corresponding author is with Institute of Informatics, Gdańsk University of Technology, Wita Stwosza 57, 80-952 Gdańsk, Poland, e-mail: hanna@inf.ug.edu.pl. A. Jastrzębski and M. Kubale are with Department of Algorithms and System Modeling, Gdańsk University of Technology, Narutowicza 11/12, 80-233 Gdańsk, Poland.

Project has been partially supported by Narodowe Centrum Nauki under contract DEC2011/02/A/ST6/00201

Received 12.04.2016. Revised 16.08.2016. 
performed, and an edge connects two tasks that should not be performed at the same time because they use the same nonsharable resource. A coloring of this graph represents a partition of tasks into subsets that may be performed simultaneously. Due to load balancing considerations, it is desirable to perform equal or nearly-equal numbers of tasks in each time slot, and this balancing is exactly what an equitable coloring achieves. Furmańczyk [11] mentions a specific application of this type of scheduling problem, namely assigning university courses to time slots in a way that avoids scheduling incompatible pairs of courses at the same time and spreads the courses evenly among the available time slots, since then the usage of scarce additional resources (e.g. rooms) is maximalized (for an alternative chromatic model see [25]).

There are very few papers on the complexity of equitable coloring. First of all, a straightforward reduction from graph coloring to equitable coloring by adding sufficiently many isolated vertices to a graph, proves that it is NP-complete to test whether a general graph has an equitable coloring with a given number of colors (greater than two). Secondly, Bodleander and Fomin [2] showed that equitable coloring can be solved to optimality in polynomial time for trees (the fact previously known due to Chen and Lih [3]) and outerplanar graphs. A polynomial time algorithm is also known for equitable coloring of split graphs [6].

This paper consists of two parts. In the first part (Section 2) we survey recent theo-

rems and conjectures concerning sufficient conditions under which a graph is equitably $r$-colorable. In addition to this we survey recent theorems and conjectures concerning the complexity of optimal equitable coloring of certain simplified families of graphs. In the second part (Section 3) we report on practical experiments with some heuristics for optimal equitable coloring of general graphs. On the basis of these experiments we claim that the best results are obtained by using the SLF coloring algorithm with the FJK balancing procedure. We state a conjecture that this heuristic algorithm produces solutions which are almost surely not worse than $(2+\varepsilon) \chi_{=}(G)$ for any $\varepsilon>0$.

\section{Recent results and conjectures}

The notion of equitable colorability was introduced by Meyer [30]. However, an earlier work of Hajnal and Szemerédi [17] showed that a graph $G$ with maximal degree $\Delta$ is equitably $k$-colorable if $k \geqslant \Delta+1$. In the same paper [30] he formulated the following conjecture:

Conjecture 1 (Equitable Coloring Conjecture (ECC)) For any connected graph G, other than complete graph or odd cycle, $\chi=(G) \leqslant \Delta$.

This conjecture has been verified for all graphs on six or fewer vertices. Lih and $\mathrm{Wu}$ [27] proved that the Equitable Coloring Conjecture is true for all bipartite graphs. Wang and Zhang [33] considered a broader class of graphs, namely $r$-partite graphs. 
They proved that Meyer's conjecture is true for complete graphs from this class. In 1994, Chen et al. [5] proposed another conjecture.

Conjecture 2 (Equitable $\Delta$-Coloring Conjecture ( $\mathbf{E} \Delta \mathbf{C C})$ ) Let $G$ be a connected graph. If $G$ is different from the complete graph, odd cycle or the complete bipartite graph $K_{\Delta, \Delta}$, then $G$ is equitably $\Delta$-colorable.

The conjecture was confirmed for outerplanar graphs [35] and planar graphs with maximum degree at least 13 [36]. The last result was improved by Nakprasit [31]. She proved that the $\mathrm{E} \Delta \mathrm{CC}$ Conjecture holds for every planar graph with maximum degree at least 9. Earlier, Zhu and $\mathrm{Bu}$ [37] proved that the Conjecture 2 holds for every $C_{3}$-free planar graph with maximum degree at least 8 and for every planar graph without $C_{4}$ and $C_{5}$ with maximum degree at least 7 . The last result was improved in [32].

Theorem 1 [32] Each $C_{3}$-free planar graph $G$ with maximum degree $\Delta \geqslant 6$ has an equitable $\Delta$-coloring.

Theorem 2 [32] Each $C_{4}$-free planar graph $G$ with maximum degree $\Delta \geqslant 7$ has an equitable $\Delta$-coloring.

Moreover, they proved that the conjecture holds for every planar graph of girth at least 6 with maximum degree at least 5 .

In 1964 Erdös [8] conjectured that any graph $G$ with maximum degree $\Delta \leqslant k$ has an equitable $(k+1)$-coloring. This conjecture was proved in 1970 by Hajnal and Szemerédi [17] with a long and complicated proof. Kierstead et al. [23] found a polynomial-time algorithm of complexity $O\left(\Delta|V(G)|^{2}\right)$ for such a coloring. Kierstead and Kostochka [21] gave a short proof of this theorem, and presented another polynomial-time algorithm for such a coloring. Hajnal-Szemerédi's bound has interesting applications in extremal combinatorial and probabilistic problems, see e.g. [1,18].

Hajnal-Szemerédi's bound, also named Dirac-type result was extended in [20]. They proved the following Ore-type theorem.

Theorem 3 [20] Every graph satisfying $\operatorname{deg}(x)+\operatorname{deg}(y) \leqslant 2 r+1$ for every edge $\{x, y\}$, has an equitable $(r+1)$-coloring.

Above theorem, like the Hajnal-Szemerédi theorem, is tight. Of course, these graphs for which the Hajnal-Szemerédi Theorem is tight simultaneously show that Theorem 3 is tight. However, there are more graphs for which this theorem is tight. For example, for every odd $n<\Delta+1$, the graph $K_{n, 2 \Delta+2-n}$ satisfies the inequality $\operatorname{deg}(x)+\operatorname{deg}(y) \leqslant$ $2 \Delta+2$ for every edge $\{x, y\}$ and has no equitable $(\Delta+1)$-coloring. The authors of [20] conjectured the following hypothesis:

Conjecture 3 [20] Let $r \geqslant 3$. If $G$ is a graph satisfying $\operatorname{deg}(x)+\operatorname{deg}(y) \leqslant 2 r$ for every edge $\{x, y\}$, and $G$ has no equitable r-coloring, then $G$ contains either $K_{r+1}$ or $K_{n, 2 r-n}$ for some odd $n$. 
The conjecture was proved for $r=3$ [20].

It is natural to ask which graphs $G$ with $\Delta=r \geqslant 3$ have equitable $r$-colorings. Certainly such graphs are $r$-colorable and do not contain $K_{r+1}$.

Chen and Yen [4] gave necessary conditions for a graph $G$ (not necessarily connected) with $\Delta \geqslant \chi(G)$ to be equitably $\Delta$-colorable and proved that those necessary conditions become sufficent when $G$ is a bipartite graph, or $G$ satisfies $\Delta \geqslant|V(G)| / 3+1$, or $G$ satisfies $\Delta \leqslant 3$. They supposed that the following is true.

Conjecture 4 [4] Let $G$ be a graph with $\Delta \geqslant \chi(G)$. Then $G$ is equitably $\Delta$-colorable if and only if at least one of the following statements holds.

1. $\Delta$ is even.

2. No component or at least two components of $G$ are isomorphic to $K_{\Delta, \Delta}$.

3. Only one component of $G$ is isomorphic to $K_{\Delta, \Delta}$ and $\alpha\left(G-K_{\Delta, \Delta}\right)>\mid V(G-$ $\left.K_{\Delta, \Delta}\right) \mid / \Delta>0$

Kierstead and Kostochka [22] introduced the notion of an $r$-equitable graph, as follows

Definition 1 Graph $G$ is $r$-equitable if

1. $|V(G)|$ is divisible by $r$

2. $G$ is $r$-colorable

3. Every $r$-coloring of $G$ is equitable.

For example, $K_{r}$ is $r$-equitable.

Conjecture 5 [22] Let graph $G$ satisfy $\Delta=r \geqslant \chi(G)$. Then $G$ has no equitable $r$ coloring if and only if $r$ is odd, $G$ has a subgraph $H$ isomorphic to $K_{r, r}$ and $G-H$ is r-equitable.

Kierstead and Kostochka [22] proved that Conjecture 5 and Conjecture 2 are equivalent.

Definition 2 An $r$-equitable graph $G$ is $r$-reducible if $V(G)$ has a partition $\left\{V_{1}, \ldots, V_{t}\right\}$ into at least two parts such that the induced subgraph $G\left[V_{i}\right]$ is $r$-equitable for each $1 \leqslant$ $i \leqslant t$; otherwise $G$ is $r$-irreducible.

Of course, $K_{r}$ is $r$-irreducible. Kierstead and Kostochka [22] identified ten other $r$ irreducible graphs $(r=3,4,5)$ (see Fig. 1). They named them $r$-basic graphs.

An $r$-decomposition of $G$ is a partition $\left\{V_{1}, \ldots, V_{t}\right\}$ of $V(G)$ such that each $G\left[V_{i}\right]$ is $r$-basic. The graph $G$ is $r$-decomposable if it has an $r$-decomposition.

It turns out that terms $r$-equitable and $r$-decomposable are equivalent for graphs $G$, where $\Delta=r$ and $r$ divides $|V(G)|$. 

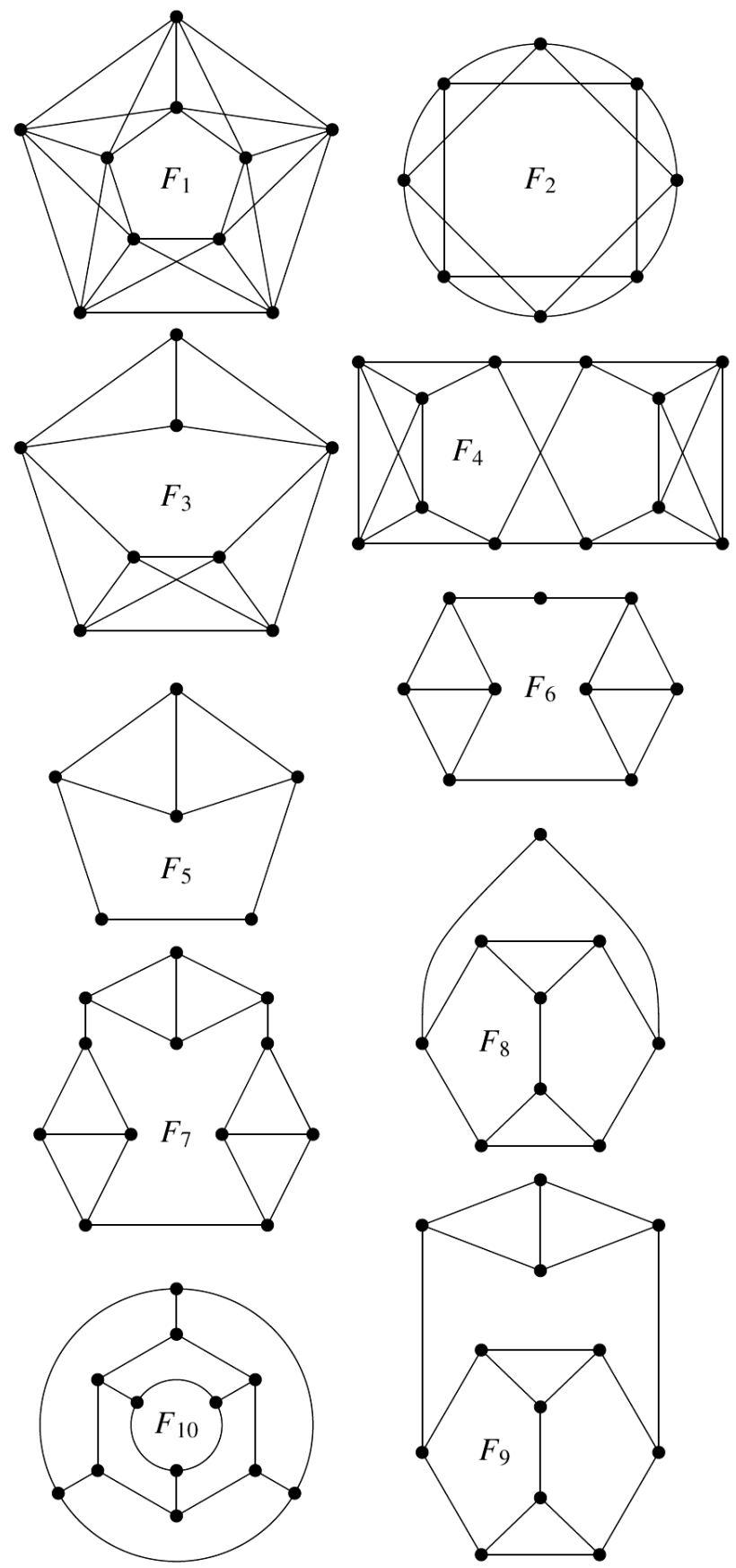

Figure 1: $r$-basic graphs; $F_{1}: r=5 ; F_{2}, F_{3}, F_{4}: r=4 ; F_{5}, \ldots F_{10}: r=3$. 
Conjecture 6 [22] Suppose that $\Delta=r \geqslant 3$ and $G$ is an $r$-colorable graph. Then $G$ is not equitably $r$-colorable if and only if the following conditions hold.

1. $r$ is odd.

2. G has a subgraph $H=K_{r, r}$.

3. $G-H$ is $r$-decomposable.

It was proved $[7,22]$ that Kierstead-Kostochka's Conjecture 6 is equivalent to the $\mathrm{E} \Delta \mathrm{CC}$ Conjecture and may help to prove it by induction.

Another research path in equitable coloring is determining exact values of equitable chromatic numbers and equitable thresholds for some specific graph classes, e.g. trees [3], complete multipartite graphs [26], Kneser graphs [9] and graph products. The last were intensively studied in $[10,11,29]$ - Cartesian products, $[11,28,34]-$ cross (Kronecker) products and in $[12,13,14,19]$ - corona products.

Among new results concerning corona products one deserves special attention. In [12] the problem for coronas of cubic graphs was considered. A cubical corona $G \circ H$ is a corona obtained by taking a cubic graph $G$ as the center graph and $|V(G)|$ copies of a cubic graph $H$ as the outer graphs. The smallest cubical corona is shown in Fig. 2. Although the problem of ordinary coloring of coronas of cubic graphs is solvable in polynomial time, the problem of equitable coloring becomes intractable for these graphs. There have been given polynomially solvable cases of coronas of cubic graphs and the general problem of colorability of cubical coronas has been proved to be NP-hard. In this way the authors established a first class of graphs for which equitable coloring problem is harder than ordinary coloring.

It is obvious that

$$
2 \leqslant \chi=(G) \leqslant 4,
$$

for any cubic graph $G$. For each $k=2,3,4$ let $C u b_{k}$ be the class of equitably $k$-chromatic cubic graphs. Let us notice that $C u b_{4}=\left\{K_{4}\right\}$. Let $C u b_{3}^{u, v, w} \subset C u b_{3}$ be the class of 3partite graphs with color classes of cardinalities $u, v$ and $w$, respectively. We have

Theorem 4 [12] The problem of deciding whether $\chi_{=}\left(K_{3,3} \circ H\right)=4$ is NP-hard even if $H \in C u b_{3}^{y, y, y}$ and $y$ is divisible by 10 .

The authors of [12] fixed all the cases of coronas of cubic graphs for which 3 colors suffice for equitable coloring. In the remaining cases they proved that 5 colors are enough for equitable coloring. These results are summarized in Tab. 1.

Moreover, a simple linear time algorithm for equitable coloring of such graphs which uses $\chi_{=}(G \circ H)$ or $\chi_{=}(G \circ H)+1$ colors was obtained. This algorithm is best possible, unless $\mathrm{P}=\mathrm{NP}$. 


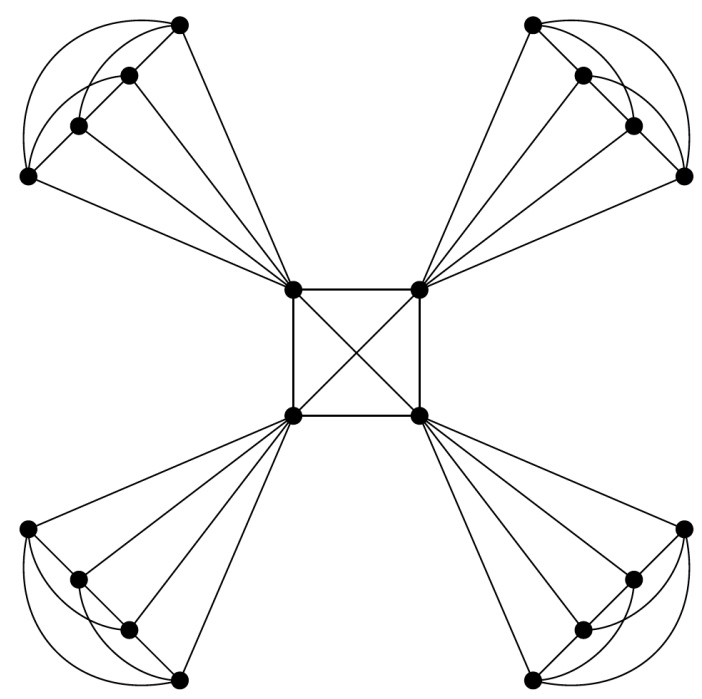

Figure 2: Corona $K_{4} \circ K_{4}$.

Table 1: Possible values of the equitable chromatic number of coronas $G \circ H$.

\begin{tabular}{|c|c|c|c|}
\hline$G$ & $\mathrm{Cub}_{2}$ & $\mathrm{Cub}_{3}$ & $\mathrm{Cub}_{4}$ \\
\hline $\mathrm{Cub} b_{2}$ & 3 or $4^{1}$ & $4 \leqslant \chi=\leqslant 5^{2}$ & 5 \\
\hline $\mathrm{Cub} b_{3}$ & 3 or $4^{1}$ & $4 \leqslant \chi=\leqslant 5^{2}$ & 5 \\
\hline $\mathrm{Cub} b_{4}$ & 4 & 4 & 5 \\
\hline
\end{tabular}

${ }^{1}$ : by this we mean that all cases when $\chi_{=}=3$ or $\chi_{=}=4$ are determined, $2:$ deciding which value $(4$ or 5$)$ is exact is NP-hard.

\section{Computer experiments}

In this section we consider two heuristics leading to an equitable coloring of a given graph. Both of them are based on heuristics for classical coloring: Greedy coloring, LF, SL, SLF [24], and in both cases our algorithms transform classical coloring into an equitable coloring. Our algorithms are based on two heuristics given in [10]. The first of them, called Naive, relies on the principle of swapping colors of vertices colored with the most and the least frequently used colors. If this failed, a new color is introduced. This algorithm has been improved by checking all possible pairs of colors of vertices colored with the most and the least frequently used colors. The new improved version, called FJK, outperforms its predecessor. 


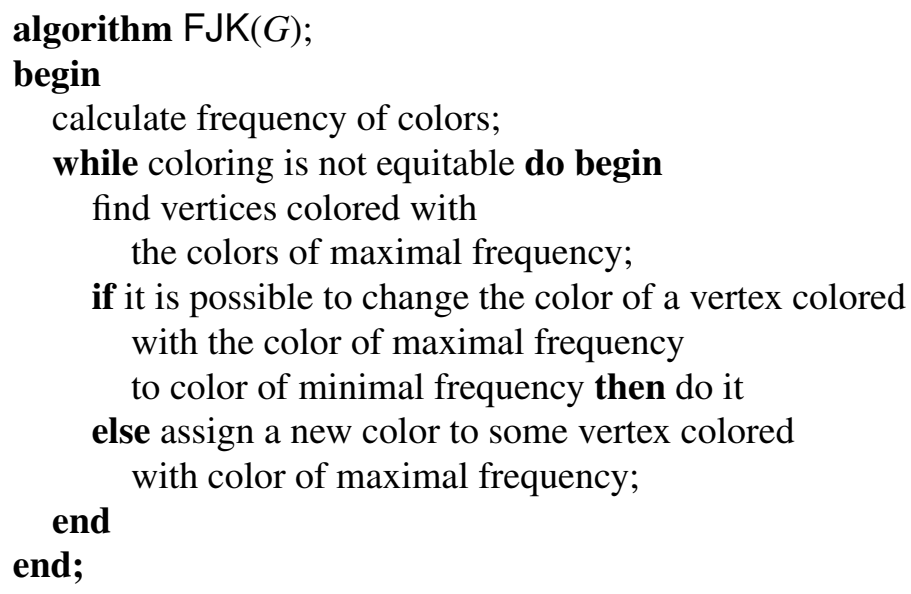

To estimate the complexity of FJK we first show that the number of recolorings in this algorithm is $O(n \log n)$. Let us consider any color class $V_{k}$. After each step of the algorithm the cardinality of $V_{k}$ may increase by 1 or decrease by 1 . The cardinality is increased if $V_{k}$ is the smallest color class. The cardinality is decreased if $V_{k}$ is the largest color class. Let sequence $\left(a_{1}^{k}, a_{2}^{k}, \ldots, a_{s}^{k}\right)$ denote the cardinalities of the color class $V_{k}$ during the course of the algorithm. It is easy to see that there exists $s_{k}$ such that for all $i<s_{k}$ we have $a_{i}^{k} \leqslant a_{i+1}^{k}$ and for all $i \geqslant s_{k}$ we have $a_{i}^{k} \geqslant a_{i+1}^{k}$. For each $i$ there cannot be more than $i$ color classes of size $\frac{n}{i}$. Therefore the maximal number of recolorings is less than

$$
\sum_{k=2}^{\frac{n}{2}} \frac{n}{k}=O(n \log n)
$$

It is easy to see that the estimation is very rough because we are not considering the graph structure neither the primary coloring. Since each recoloring takes time $O\left(n^{2}\right)$, so the overall complexity of the algorithm is $O\left(n^{3} \log n\right)$.

The second heuristic, CreateSubgraph2, is based on CreateSubgraph from [10] and relies on the idea of swapping colors in entire subgraphs induced by vertices colored with the most and the least frequently used colors. The former algorithm has been improved by checking all possible pairs of color classes with the most and the least frequently used colors. The algorithm swaps 2 -colored subgraphs optimaly, i.e. the difference between the number of vertices colored with the first color and the number of vertices with the second color is as small as possible. We skip a detailed description of CreateSubgraph2, since its results appeared worse than those of FJK.

Those two balancing procedures followed the four above-mentioned classical algorithms (in this way we obtained 8 particular algorithms). We tested them on random graphs of order $n=100,200, \ldots, 1000$ and densities $d=0.1, \ldots, 0.9$. For each fixed order $n$ and fixed density $d$ we generated 100 graphs at random. For each graph we tested each combination of the algorithms (72000 runs altogether). The tests were performed on AMD Athlon 64 X2 5000+ with 2GB of RAM. Because of the size of the evidence we present herein only part of the results. 
Table 2: Average numbers of colors used by: O - Greedy coloring, A - Greedy+ FJK algorithm and B - Greedy+CreateSubgraph2 algorithm.

\begin{tabular}{|c|c|c|c|c|c|c|c|c|c|c|c|}
\hline 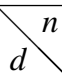 & & 100 & 200 & 300 & 400 & 500 & 600 & 700 & 800 & 900 & 1000 \\
\hline \multirow{3}{*}{0.1} & $\mathrm{O}$ & 6.25 & 9.99 & 13.08 & 581 & 1852 & 21.08 & 23.58 & 25.78 & 28.20 & 30.52 \\
\hline & A & 6.26 & 10.08 & 13.14 & 16.02 & 18.82 & 21.40 & 24.24 & 26.15 & 29.02 & 30.85 \\
\hline & B & 6.30 & 10.10 & 13.38 & 16.20 & 19.14 & 21.67 & 24.39 & 26.38 & 29.21 & 31.23 \\
\hline \multirow{3}{*}{0.2} & $\mathrm{O}$ & 9.68 & 15.98 & 21.32 & 26.36 & 31.10 & 35.67 & 39.97 & 44.41 & 48.62 & 52.68 \\
\hline & A & 10.08 & 16.31 & 22.19 & 26.82 & 31.99 & 36.45 & 41.05 & 45.35 & 50.03 & 53.70 \\
\hline & B & 10.02 & 16.46 & 22.03 & 27.16 & 31.88 & 36.51 & 41.34 & 45.70 & 50.26 & 54.27 \\
\hline \multirow{3}{*}{0.3} & $\mathrm{O}$ & 3.16 & 21.80 & 29.63 & 36.81 & 43.62 & 50.24 & 56.75 & 62.99 & 69.20 & 75.20 \\
\hline & A & 13.56 & 22.48 & 30.54 & 38.02 & 45.00 & 51.58 & 58.50 & 64.64 & 70.86 & 77.10 \\
\hline & B & 13.49 & 22.63 & 30.85 & 38.07 & 45.38 & 52.29 & 59.26 & 65.38 & 71.57 & 77.97 \\
\hline \multirow{3}{*}{0.4} & $\mathrm{O}$ & 16.55 & 28.07 & 38.13 & 47.78 & 57.21 & 65.92 & 74.52 & 82.96 & 90.76 & 99.15 \\
\hline & A & 17.27 & 29.03 & 39.29 & 49.53 & 58.55 & 67.97 & 77.15 & 85.04 & 93.19 & 101.68 \\
\hline & B & 17.14 & 29.20 & 39.86 & 50.00 & 59.05 & 68.66 & 78.05 & 86.50 & 94.55 & 103.15 \\
\hline \multirow{3}{*}{0.5} & $\mathrm{O}$ & 20.49 & 34.50 & 47.56 & 59.97 & 71.46 & 82.79 & 94.08 & 104.71 & 115.14 & 125.39 \\
\hline & A & 21.11 & 35.56 & 49.51 & 61.28 & 73.46 & 86.20 & 97.69 & 107.14 & 117.96 & 128.94 \\
\hline & B & 21.16 & 35.70 & 50.05 & 62.24 & 73.86 & 87.28 & 98.53 & 109.40 & 120.03 & 130.68 \\
\hline \multirow{3}{*}{0.6} & $\mathrm{O}$ & 24.76 & 42.27 & 58.34 & 73.57 & 88.43 & 102.57 & 116.33 & 129.89 & 143.23 & 156.01 \\
\hline & A & 25.66 & 43.34 & 60.94 & 76.30 & 90.18 & 105.30 & 119.92 & 134.93 & 150.39 & 163.47 \\
\hline & B & 25.62 & 3.83 & 61.34 & 76.93 & 92.10 & 107.02 & 121.18 & 136.76 & 152.38 & 164.96 \\
\hline \multirow{3}{*}{0.7} & $\mathrm{O}$ & 29.48 & 51.67 & 71.44 & 89.92 & 108.39 & 126.19 & 143.12 & 160.34 & 176.77 & 193.45 \\
\hline & A & 0.34 & 3.09 & 75.31 & 91.82 & 110.31 & 129.60 & 146.71 & 164.81 & 183.46 & 202.44 \\
\hline & B & 30.38 & 3.35 & 75.91 & .43 & 42 & 130.56 & 147.74 & 166.51 & 185.71 & 205.28 \\
\hline \multirow{3}{*}{0.8} & $\mathrm{O}$ & 35.60 & 62.85 & 88.19 & 112.09 & 135.00 & 157.35 & 179.08 & 200.73 & 221.43 & 242.53 \\
\hline & A & 36.37 & 66.22 & 89.87 & 113.56 & 137.73 & 160.65 & 183.17 & 206.44 & 229.93 & 254.01 \\
\hline & B & 36.45 & 66.14 & 89.94 & 114.06 & 138.82 & 161.40 & 184.35 & 208.61 & 232.59 & 256.98 \\
\hline \multirow{3}{*}{0.9} & $\mathrm{O}$ & 45.87 & 81.61 & 114.48 & 145.69 & 176.52 & 205.99 & 235.06 & 264.19 & 291.76 & 319.83 \\
\hline & A & 47.63 & 81.84 & 115.66 & 147.67 & 179.20 & 210.14 & 241.69 & 273.55 & 305.92 & 338.21 \\
\hline & B & 47.37 & 81.84 & 115.99 & 147.53 & 179.91 & 211.30 & 243.59 & 276.47 & 309.24 & 342.05 \\
\hline
\end{tabular}

In Tabs. 2 and 3 we present average number of colors obtained by the algorithms. The main classical coloring is denoted by O, the FJK algorithm by A and the CreateSubgraph2 algorithm by B. As one can see, in most cases, the second algorithm does not give better result and, moreover, it runs in a longer time (cf. Tab. 4). Therefore, we consider only the FJK algorithm in the further part of this section. Moreover, the best results were obtained when the balancing procedure followed the SLF algorithm. Therefore, in Tab. 3 we report on computational results involving SLF. 
Table 3: Average numbers of colors used by: O - SLF algorithm, A - SLF+FJK algorithm and B - SLF+CreateSubgraph2 algorithm.

\begin{tabular}{|c|c|c|c|c|c|c|c|c|c|c|c|}
\hline 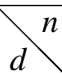 & & 100 & 200 & 300 & 400 & 500 & 600 & 700 & 800 & 900 & 1000 \\
\hline \multirow{3}{*}{0.1} & $\mathrm{O}$ & 4.64 & 7.43 & 10.05 & 238 & 1482 & 17.03 & 19.12 & 21.21 & 23.23 & 25.37 \\
\hline & A & 4.93 & 7.92 & 10.83 & 13.41 & 15.80 & 18.61 & 20.92 & 23.08 & 25.69 & 28.01 \\
\hline & B & 5.07 & 8.28 & 11.28 & 13.64 & 16.43 & 19.10 & 21.30 & 23.79 & 26.23 & 27.92 \\
\hline \multirow{3}{*}{0.2} & $\mathrm{O}$ & 7.77 & 12.71 & 17.56 & 2106 & 26.25 & 30.43 & 34.46 & 38.30 & 42.21 & 45.93 \\
\hline & A & 8.39 & 13.83 & 19.59 & 24.37 & 29.13 & 33.42 & 37.50 & 41.50 & 45.63 & 49.58 \\
\hline & B & 8.61 & 14.03 & 19.80 & 24.78 & 29.44 & 33.88 & 38.16 & 42.88 & 47.04 & 51.16 \\
\hline \multirow{3}{*}{0.3} & $\mathrm{O}$ & 10.56 & 18.15 & 25.10 & 31.72 & 37.98 & 43.98 & 49.92 & 55.64 & 61.33 & 67.02 \\
\hline & A & 11.39 & 19.98 & 27.20 & 34.25 & 41.01 & 47.24 & 53.50 & 59.56 & 65.63 & 71.93 \\
\hline & B & 11.73 & 20.48 & 28.01 & 35.51 & 42.12 & 49.12 & 55.45 & 61.72 & 68.24 & 74.14 \\
\hline \multirow{3}{*}{0.4} & $\mathrm{O}$ & 13.85 & 23.83 & 33.12 & 41.90 & 50.26 & 58.37 & 66.52 & 74.39 & 81.93 & 89.43 \\
\hline & A & 15.11 & 25.67 & 35.48 & 44.90 & 53.88 & 62.15 & 71.59 & 79.83 & 87.49 & 94.29 \\
\hline & B & 15.43 & 26.77 & 36.85 & 46.46 & 55.95 & 65.45 & 73.12 & 82.10 & 90.62 & 98.55 \\
\hline \multirow{3}{*}{0.5} & $\mathrm{O}$ & 17.19 & 30.11 & 41.92 & 53.20 & 64.10 & 74.63 & 85.01 & 94.98 & 104.93 & 114.60 \\
\hline & A & 18.56 & 32.31 & 44.50 & 57.32 & 68.39 & 78.86 & 89.63 & 101.45 & 113.20 & 122.74 \\
\hline & B & 18.59 & 33.17 & 45.98 & 58.29 & 70.72 & 82.53 & 92.48 & 103.82 & 116.27 & 127.22 \\
\hline \multirow{3}{*}{0.6} & $\mathrm{O}$ & 21.26 & 37.25 & 52.08 & 66.18 & 79.97 & 93.16 & 105.81 & 118.89 & 131.19 & 143.63 \\
\hline & A & 22.61 & 40.16 & 54.83 & 69.65 & 85.17 & 100.97 & 114.24 & 126.13 & 138.30 & 150.84 \\
\hline & B & 22.74 & 1.09 & 56.23 & 70.95 & 86.90 & 103.20 & 117.53 & 131.08 & 143.23 & 154.96 \\
\hline \multirow{3}{*}{0.7} & $\mathrm{O}$ & 25.71 & 45.81 & 64.38 & 82.17 & 99.25 & 115.32 & 131.66 & 147.68 & 163.76 & 179.16 \\
\hline & A & 26.75 & 9.45 & 67.26 & 85.68 & 103.83 & 122.88 & 142.37 & 160.55 & 174.93 & 191.44 \\
\hline & B & 26.95 & 0.46 & 68.50 & .92 & 106.05 & 125.45 & 145.06 & 163.65 & 181.05 & 196.94 \\
\hline \multirow{3}{*}{0.8} & $\mathrm{O}$ & 31.88 & 56.89 & 80.33 & 102.71 & 124.37 & 145.23 & 165.43 & 186.23 & 206.10 & 225.88 \\
\hline & A & 33.94 & 58.30 & 82.50 & 106.17 & 129.95 & 153.84 & 178.55 & 201.37 & 222.34 & 240.67 \\
\hline & B & 34.29 & 58.63 & 83.52 & 107.47 & 131.94 & 156.79 & 181.15 & 205.22 & 225.75 & 243.74 \\
\hline \multirow{3}{*}{0.9} & $\mathrm{O}$ & 41.45 & 74.80 & 105.01 & 133.89 & 162.75 & 190.99 & 219.52 & 246.72 & 273.96 & 300.87 \\
\hline & A & 41.63 & 75.89 & 107.44 & 139.25 & 171.71 & 204.44 & 236.16 & 263.28 & 284.75 & 308.47 \\
\hline & B & 41.63 & 76.07 & 107.98 & 141.13 & 174.37 & 207.94 & 239.92 & 265.71 & 284.55 & 307.00 \\
\hline
\end{tabular}

Also, we compared results obtained by Greedy (classical coloring) and SLF+FJK (equitable coloring) algorithms. The results are summarized in Tab. 5 and two columns of it $(d=0.1$ and $d=0.5)$ are shown in Fig. 3. We have chosen these graph densities because graphs encountered in real-life applications are rather sparse. In $88 \%$ of cases the number of colors used by the latter is not greater than those by Greedy coloring. In the remaining $12 \%$ cases the number of colors used by SLF+FJK exceeds that of Greedy coloring by at most $20 \%$, and the average is $3,8 \%$. 
Table 4: Average computation time [ms] of: O - SLF algorithm, A - SLF+FJK algorithm and B - SLF+CreateSubgraph2 algorithm.

\begin{tabular}{|c|c|c|c|c|c|c|c|c|c|c|c|}
\hline$d$ & & 100 & 200 & 300 & 400 & 500 & 600 & 700 & 800 & 900 & 1000 \\
\hline \multirow{2}{*}{0.1} & A & 0 & 1 & 3 & 4 & 8 & 13 & 21 & 29 & 47 & 60 \\
\hline & B & 5 & 21 & 49 & 81 & 140 & 237 & 329 & 464 & 657 & 770 \\
\hline \multirow{2}{*}{0.2} & A & 0 & 1 & 5 & 10 & 19 & 30 & 43 & 65 & 89 & 118 \\
\hline & B & 8 & 28 & 99 & 198 & 334 & 529 & 787 & 1195 & 1639 & 2128 \\
\hline \multirow{2}{*}{0.3} & A & 0 & 2 & 7 & 14 & 24 & 42 & 64 & 89 & 131 & 179 \\
\hline & B & 10 & 53 & 144 & 301 & 550 & 945 & 1358 & 1991 & 2784 & 3637 \\
\hline \multirow{2}{*}{0.4} & A & 0 & 2 & 8 & 17 & 33 & 50 & 92 & 130 & 178 & 199 \\
\hline & B & 12 & 76 & 198 & 406 & 778 & 1411 & 1981 & 2876 & 3973 & 5069 \\
\hline \multirow{2}{*}{0.5} & A & 1 & 3 & 8 & 23 & 40 & 56 & 84 & 156 & 254 & 323 \\
\hline & B & 13 & 86 & 241 & 546 & 996 & 1715 & 2382 & 3876 & 6023 & 8046 \\
\hline \multirow{2}{*}{0.6} & A & 0 & 4 & 8 & 19 & 44 & 100 & 152 & 168 & 199 & 238 \\
\hline & B & 13 & 112 & 246 & 561 & 1300 & 2726 & 3965 & 5023 & 6544 & 7128 \\
\hline \multirow{2}{*}{0.7} & A & 0 & 4 & 9 & 16 & 34 & 92 & 171 & 281 & 341 & 452 \\
\hline & B & 12 & 131 & 259 & 558 & 1514 & 3500 & 6169 & 8638 & 11724 & 14324 \\
\hline \multirow{2}{*}{0.8} & A & 0 & 2 & 6 & 13 & 33 & 89 & 180 & 302 & 432 & 526 \\
\hline & B & 20 & 53 & 214 & 770 & 2243 & 4996 & 8966 & 13122 & 16575 & 16852 \\
\hline \multirow{2}{*}{0.9} & A & 0 & 1 & 3 & 14 & 41 & 108 & 184 & 281 & 283 & 290 \\
\hline & B & 5 & 58 & 385 & 1552 & 4013 & 8142 & 11963 & 14308 & 11196 & 7356 \\
\hline
\end{tabular}

It is known that algorithm Greedy is practically 2-approximate. More precisely, Grimmet and McDiarmid [16] showed that the Greedy algorithm when applied to a random ordering of the vertices yields a $\operatorname{Greedy}(G)$-coloring for any $\varepsilon>0$, where

$$
\operatorname{Greedy}(G) \leqslant(2+\varepsilon) \chi(G)
$$

for all but a vanishingly small fraction of graphs as the number of vertices tends to infinity.

On the basis of experimental results in general and Fig. 3 in particular we conjecture that our FJK algorithm yields the same guarantee.

\section{Conjecture 7}

$$
\operatorname{SLF}+\mathrm{FJK}(G) \leqslant(2+\varepsilon) \chi=(G)
$$

for almost every graph $G$.

All the algorithms used in tests can be found in the KOALA library [15]. 
Table 5: Greedy vs SLF+FJK algorithm. The notation $X / Y / Z$ denotes that in $X$ cases Greedy algorithm used fewer colors, in $Y$ cases the both algorithms used the same number of colors and in $Z$ cases the algorithm SLF+FJK was better.

\begin{tabular}{|c|c|c|c|c|c|c|c|c|c|}
\hline$n^{d}$ & 0.1 & 0.2 & 0.3 & 0.4 & 0.5 & 0.6 & 0.7 & 0.8 & 0.9 \\
\hline 100 & $1 / 8 / 91$ & $6 / 14 / 80$ & $4 / 8 / 88$ & $11 / 10 / 79$ & $7 / 15 / 78$ & $14 / 7 / 79$ & $1 / 5 / 94$ & $12 / 11 / 77$ & $1 / 1 / 98$ \\
\hline 200 & $0 / 3 / 97$ & $8 / 3 / 89$ & $5 / 8 / 87$ & $5 / 4 / 91$ & $7 / 20 / 73$ & $5 / 18 / 77$ & $12 / 14 / 74$ & 9/ 0/91 & $0 / 0 / 100$ \\
\hline 300 & $0 / 6 / 94$ & $5 / 11 / 84$ & $3 / 4 / 93$ & $7 / 12 / 81$ & $2 / 2 / 96$ & $14 / 4 / 82$ & $13 / 0 / 87$ & $2 / 1 / 97$ & $0 / 0 / 100$ \\
\hline 400 & $5 / 6 / 89$ & $2 / 8 / 90$ & $4 / 3 / 93$ & $2 / 0 / 98$ & $4 / 5 / 91$ & $1 / 3 / 96$ & $4 / 1 / 95$ & $1 / 1 / 98$ & $2 / 0 / 98$ \\
\hline 500 & $0 / 4 / 96$ & $3 / 15 / 82$ & $1 / 6 / 93$ & $4 / 10 / 86$ & $21 / 8 / 71$ & $3 / 5 / 92$ & $2 / 0 / 98$ & $0 / 3 / 97$ & $2 / 2 / 96$ \\
\hline 600 & 2/7/91 & 4/ 5/91 & $1 / 7 / 92$ & $9 / 1 / 90$ & 9/ 0/91 & $13 / 21 / 66$ & $1 / 4 / 95$ & $2 / 7 / 91$ & $24 / 10 / 66$ \\
\hline 700 & $0 / 5 / 95$ & $0 / 9 / 91$ & $0 / 0 / 100$ & $8 / 3 / 89$ & $1 / 1 / 98$ & $48 / 8 / 44$ & $22 / 27 / 51$ & $37 / 13 / 50$ & $74 / 8 / 18$ \\
\hline 800 & $4 / 7 / 89$ & $0 / 4 / 96$ & $0 / 11 / 89$ & $3 / 6 / 91$ & $6 / 4 / 90$ & $31 / 0 / 69$ & $69 / 10 / 21$ & $67 / 6 / 27$ & $68 / 0 / 32$ \\
\hline 900 & $5 / 4 / 91$ & $0 / 3 / 97$ & $3 / 9 / 88$ & $10 / 17 / 73$ & $4 / 17 / 79$ & $15 / 1 / 84$ & $56 / 0 / 44$ & $73 / 0 / 27$ & $34 / 0 / 66$ \\
\hline 1000 & $1 / 3 / 96$ & $5 / 2 / 93$ & $5 / 2 / 93$ & 9/ 0/91 & $35 / 14 / 51$ & $5 / 1 / 94$ & $46 / 0 / 54$ & $51 / 0 / 49$ & $19 / 0 / 81$ \\
\hline
\end{tabular}

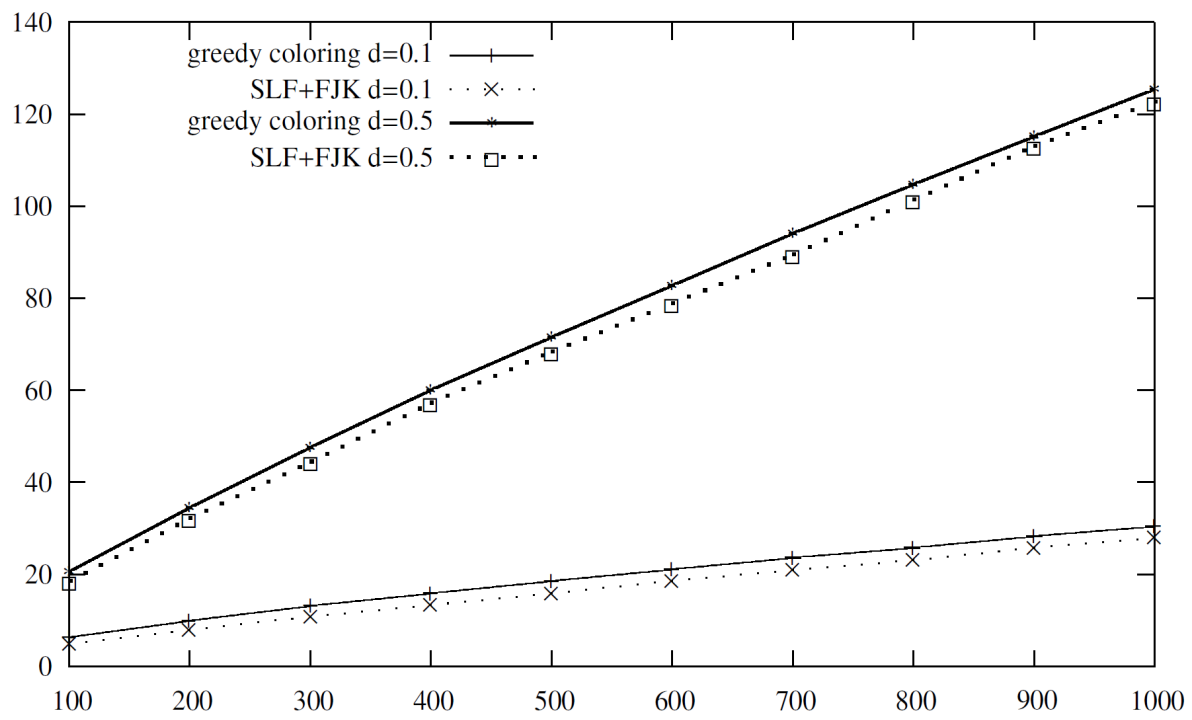

Figure 3: Greedy vs SLF+FJK algorithms - avarage numbers of colors used.

\section{Final remarks}

An important branch of scheduling theory is chromatic scheduling. In this model we have a number of identical tasks represented by graph vertices on one hand and a number of conflicts between tasks modeled by graph edges, on the other. Our aim is to assign tasks to time slots, i.e. to construct a legal schedule of length as small as possible. 
This aim can be accomplished by coloring the vertices of underlying graph, since such a coloring represents a partition of tasks into subsets that can be performed simultaneously. However, because of load balancing constraints, it is necessary to perform equal or nearly-equal numbers of tasks in each time slot. In other words, our solution must be not only frugal with colors but equitable as well. We know that efficient constructing such a solution is difficult, since the problem is intractable. However, if we relax our scheduling problem by allowing more colors than the equitable chromatic number and apply the SLF+FJK heuristic algorithm then we will be almost sure to know that our solution is not worse than twice $C_{\max }^{*}$, where $C_{\max }^{*}$ is the length of an optimal schedule.

\section{References}

[1] N. AlON and R. YUSTER: $H$-factors in dense graphs. J. Combin. Theory Ser. B, 66 (1996), 269-282.

[2] H.L. Bodleander and F.V. Fomin: Equitable colorings of bounded treewidth graphs. Theor. Comput. Sci., 349(1), (2005), 22-30.

[3] B.L. Chen and K.W. LiH: Equitable coloring of trees. J. Combin. Theory Ser. B, 61 (1994), 83-87.

[4] B.L. Chen and Ch.H. Yen: Equitable $\Delta$-coloring of graphs. Disc. Math., 312 (2012), 1512-1517.

[5] B.L. CHEN, K.W. LIH and P.L. WU: Equitable coloring and the maximum degree. Europ. J. Combinatorics, 15 (1994), 443-447.

[6] B.L. Chen, M.T. Ko and K.W. LiH: Equitable and $m$-bounded coloring of split graphs. In Combinatorics and Computer Science, LCNS 1120, Springer, 1995.

[7] B.L. Chen, K.W. LIH and CH.H. Yen: Equivalence of two conjectures on equitable coloring of graphs. J. Combinatorial Optimization, 25(4), (2013), 501-504.

[8] P. Erdös. Problem 9. In M. Fielder, editor, Theory of Graphs and its Applications. Czech. Acad. Sci. Publ., Prague, 1964. Vol. 159.

[9] R. FidyteK, H. FURMAŃCZYK and P. ŻYLIŃSKi: Equitable coloring of Kneser graphs. Discussiones Mathematicae Graph Theory, 29(1), (2009), 119-142.

[10] H. FURMAŃCZYK: Graph Colorings, chapter Equitable coloring of graphs. American Mathematical Society Providence, Rhode Island, 2004.

[11] H. FURMAŃCZYK: Equitable coloring of graph products. Opuscula Mathematica, 26(1), (2006), 31-44. 
[12] H. FurmańCZyK and M. Kubale: Equitable coloring of corona products of cubic graphs is harder than ordinary coloring. Ars Mathematica Contemporanea, 10(2), (2016), 333-347.

[13] H. Furmańczyk, M. Kubale and V. Mkrtchyan: Equitable colorings of corona multiproducts of graphs. arXiv:1210.6568, 2012.

[14] H. Furmańczy K, K. Kaliraj, M. Kubale and V.J. Vivin: Equitable coloring of corona products of graphs. Adv. Appl. Disc. Math., 11(2), (2013), 103-120.

[15] T. Goluch, K.M. OcetKiewicz and K. Giaro: Koala graph theory internet service. TASK Quarterly, 19(4), (2015), 455-470.

[16] G. R. Grimmet and C. MCDiarmid: On coloring random graphs. Math. Proc. Cambridge Philos. Soc., 77 (1975), 313-324.

[17] A. HAJnAl and E. Szemerédi: Proof of a conjecture of Erdos. In Combinatorial Theory and Its Applications, II. Colloq. Math. Soc. Janos Bolyai, Vol. 4, NorthHolland, Amsterdam, 1970.

[18] S. JANSON and A. RUCIŃSKI: The infamous upper tail. Random Structures and Algorithms, 20 (2001), 317-342.

[19] K. KaliRaJ, V.J. Vivin and V.J. ViVIK: Equitable coloring on corona graph of graphs. J. Comb. Math. Comb. Comput., 81 (2012), 191-197.

[20] H.A. Kierstead and A.V. Kostochka: An Ore-type theorem on equitable coloring. J. Combin. Theory Ser. B, 98 (2008), 226-234.

[21] H.A. Kierstead and A.V. KostochKa: A short proof of the Hajnal-Szemeredi theorem on equitable colouring. Combinatorics, Probability and Computing, 17(2), (2008), 265-270.

[22] H.A. Kierstead and A.V. KostochKa: Equitable versus nearly equitable coloring and the Chen-Lih-Wu conjecture. Combinatorica, 30(2), (2010), 201-216.

[23] H.A. Kierstead, A.V. Kostochka, M. Mydlarz and E. Szemerédi: A fast algorithm for equitable coloring. Combinatorica, 30(2), (2010), 217-224.

[24] A. Kosowski and K. MAnUszewski: Graph Colorings, chapter Classical coloring of graphs. American Mathematical Society Providence, Rhode Island, 2004.

[25] M. Kubale: Interval vertex-coloring of a graph with forbidden colors. Disc. Math., 74, (1989), 125-136.

[26] P.C.B. LAM, W.C. SHIU, C.S. Tong and C. F. ZHANG: On the equitable chromatic number of complete $n$-partite graphs. Disc. Appl. Math., 113(2-3), (2001), 307-310. 
[27] K.W. LIH and P.L. WU: On equitable coloring of bipartite graphs. Disc. Math., 151 (1996), 155-160.

[28] W.-H. LIN and G.J. ChANG: Equitable colorings of Kronecker products of graphs. Disc. Appl. Math., 158 (2010), 1816-1826.

[29] W.-H. LIN and G.J. CHANG: Equitable colorings of Cartesian products of graphs. Disc. Appl. Math., 160 (2012), 239-247.

[30] W. Meyer: Equitable coloring. Amer. Math. Monthly, 80 (1973), 920-922.

[31] K. NAKPRASIT: Equitable colorings of planar graphs with maximum degree at least nine. Disc. Math., 312 (2012), 1019-1024.

[32] K. NAKPRASIT and K. NAKPRASIT: Equitable colorings of planar graphs without short cycles. Theoret. Comput. Sci., 465 (2012), 21-27.

[33] W. WANG and K. ZHANG: Equitable colorings of line graphs and complete $r$ partite graphs. Systems Science and Mathematical Sciences, 13(2), (2000), 190194 ,

[34] Z.D. YAN and W. WANG: Equitable chromatic threshold of Kronecker products of complete graphs. arXiv:1208.0918v3, 2012.

[35] H.P. YAP and Y. ZHANG: The equitable $\Delta$-coloring conjecture holds for outerplanar graphs. Bulletin of the Inst. of Math. Academia Sinica, 25 (1997), 143-149.

[36] H.P. YAP and Y. ZHANG: Equitable colourings of planar graphs. J. Comb. Math. Comb. Comput., 27 (1998), 97-105.

[37] J. ZHU and Y. BU: Equitable list colorings of planar graphs without short cycles. Theoret. Comput. Sci., 407 (2008), 21-28. 